\title{
ON THE SPECTRAL CHARACTERIZATIONS OF GRAPHS
}

\author{
Jing Huang AND Shuchao Li \\ Faculty of Mathematics and Statistics \\ Central China Normal University \\ Wuhan 430079, P.R. China \\ e-mail: 1042833291@qq.com (J. Huang) \\ lscmath@mail.ccnu.edu.cn (S.C. Li)
}

\begin{abstract}
Several matrices can be associated to a graph, such as the adjacency matrix or the Laplacian matrix. The spectrum of these matrices gives some informations about the structure of the graph and the question "Which graphs are determined by their spectrum?" is still a difficult problem in spectral graph theory. Let $\mathscr{U}_{p}^{2 q}$ be the set of graphs obtained from $C_{p}$ by attaching two pendant edges to each of $q(q \leqslant p)$ vertices on $C_{p}$, whereas $\mathscr{V}_{p}^{2 q}$ the subset of $\mathscr{U}_{p}^{2 q}$ with odd $p$ and its $q$ vertices of degree 4 being nonadjacent to each other. In this paper, we show that each graph in $\mathscr{U}_{p}^{2 q}, p$ even and its $q$ vertices of degree 4 being consecutive, is determined by its Laplacian spectrum. As well we show that if $G$ is a graph without isolated vertices and adjacency cospectral with the graph in $\mathscr{V}_{p}^{p-1}=\{H\}$, then $G \cong H$.
\end{abstract}

Keywords: Laplacian spectrum, adjacency spectrum, cospectral graphs, spectral characterization.

2010 Mathematics Subject Classification: 05C50, 15A18.

\section{REFERENCES}

[1] S. Barik, S. Patiand and B.K. Sarma, The spectrum of the corona of two graphs, SIAM J. Discrete Math. 21 (2007) 47-56.

doi:10.1137/050624029

[2] J.A. Bondy and U.S.R. Murty, Graph Theory, in: Graduate Texts in Mathematics, 244 (Springer, 2008).

[3] R. Boulet, Spectral characterization of sun graphs and broken sun graphs, Discrete Math. Theor. Comput. Sci. 11 (2009) 149-160. 
[4] C.J. Bu and J. Zhou, Laplacian spectra characterization of some graphs obtained by product operation, Discrete Math. 312 (2012) 1591-1595. doi:10.1016/j.disc.2012.02.002

[5] C.J. Bu, J. Zhou, H.B. Li and W.Z. Wang, Spectral characterization of the corona of a cycle and two isolated vertices, Graphs Combin. 30 (2014) 1123-1133. doi:10.1007/s00373-013-1327-7

[6] L. Collatz and U. Sinogowitz, Spektren endlicher Grafen, Abh. Math. Semin. Univ. Hambg. 21 (1957) 63-77. doi:10.1007/BF02941924

[7] D. Cvetković, M. Doob and H. Sachs, Spectra of Graphs: Theory and Applications (Academic Press, New York, San Francisco, London, 1980).

[8] D. Cvetković and P. Rowlinson, Spectra of unicyclic graphs, Graphs Combin. 3 (1987) 7-23. doi:10.1007/BF01788525

[9] M. Cámara and W.H. Haemers, Spectral characterizations of almost complete graphs, Discrete Appl. Math. 176 (2014) 19-23. doi:10.1016/j.dam.2013.08.002

[10] E.R. van Dam and W.H. Haemers, Which graphs are determined by their spectrum? Linear Algebra Appl. 373 (2003) 241-272. doi:10.1016/S0024-3795(03)00483-X

[11] M. Doob, Eigenvalues of graphs, in: L.W. Beineke, R.J. Wilson (Eds.), Topics in Algebraic Graph Theory (Cambridge University Press, 2005).

[12] O. Favaron, M. Mahéo, and J.F. Saclé, Some eigenvalues properties in graphs, Discrete Math. 111 (1993) 197-200.

doi:10.1016/0012-365X(93)90156-N

[13] N. Ghareghai, G.R. Omidi and B. Tayfeh-Rezaie, Spectral characterization of graphs with index at most $\sqrt{2+\sqrt{5}}$, Linear Algebra Appl. 420 (2007) 483-489. doi:10.1016/j.laa.2006.08.009

[14] C. Godsil and G. Royle, Algebraic Graph Theory (Springer, 2001). doi:10.1007/978-1-4613-0163-9

[15] A.K. Kelmans and V.M. Chelnokov, A certain polynomial of a graph and graphs with an extremal number of trees, J. Combin. Theory Ser. B 16 (1974) 197-214. doi:10.1016/0095-8956(74)90065-3

[16] J.S. Li and X.D. Zhang, On the Laplacian eigenvalues of a graph, Linear Algebra Appl. 285 (1998) 305-307. doi:10.1016/S0024-3795(98)10149-0

[17] F.J. Liu, Q.X. Huang, J.F. Wang and Q.H. Liu, The spectral characterization of $\infty$-graphs, Linear Algebra Appl. 437 (2012) 1482-1502. doi:10.1016/j.laa.2012.04.013 
[18] M. Mirzakhah and D. Kiani, The sun graph is determined by its signless Laplacian spectrum, Electron. J. Linear Algebra. 20 (2010) 610-620.

doi:10.13001/1081-3810.1397

[19] G.R. Omidi and K. Tajbakhsh, Starlike trees are determined by their Laplacian spectrum, Linear Algebra Appl. 422 (2007) 654-658.

doi:10.1016/j.laa.2006.11.028

[20] V.M. Piet, Graph Spectra for Complex Networks (Cambridge University Press, 2010).

[21] X.L. Shen, Y.P. Hou and Y.P. Zhang, Graph $Z_{n}$ and some graphs related to $Z_{n}$ are determined by their spectrum, Linear Algebra Appl. 404 (2005) 58-68. doi:10.1016/j.laa.2005.01.036

[22] W. Wang and C.X. Xu, On the spectral characterization of T-shape trees, Linear Algebra Appl. 414 (2006) 492-501. doi:10.1016/j.laa.2005.10.031

[23] W. Wang and C.X. Xu, The T-shape tree is determined by its Laplacian spectrum, Linear Algebra Appl. 419 (2006) 78-81.

doi:10.1016/j.laa.2006.04.005

Received 2 January 2015

Revised 13 June 2016

Accepted 13 June 2016 